\title{
A short proof of the formula of Faà di Bruno
}

\author{
Karlheinz Spindler \\ Karlheinz Spindler received his mathematical education at the Technische Hochschule \\ Darmstadt. After obtaining his Ph.D. in mathematics, he spent two years as a visit- \\ ing assistant professor at Louisiana State University in Baton Rouge (USA) and then \\ worked for five years in the Flight Dynamics Department of the European Space Op- \\ erations Centre (ESOC) in Darmstadt. At present, he teaches mathematics and data \\ processing at the Fachhochschule Wiesbaden. His research interests include geomet- \\ ric methods in control theory and parameter estimation methods related to the study \\ of dynamical systems.
}

While Leibniz' formula $(f g)^{(n)}=\sum_{k=0}^{n}\left(\begin{array}{l}n \\ k\end{array}\right) f^{(k)} g^{(n-k)}$ for the higher-order derivatives of the product of two functions is common mathematical knowledge, its analogue for the composition of two functions is much less well known.

Formula of Faà di Bruno. If $f$ and $g$ possess derivatives up to order $n$, then

$(f \circ g)^{(n)}=\sum_{k=0}^{n} \sum_{\substack{k_{1}+k_{2}+\cdots+k_{n}=k, k_{1}+2 k_{2}+\cdots+n k_{n}=n}} \frac{n !}{k_{1} ! k_{2} ! \cdots k_{n} !}\left(f^{(k)} \circ g\right)\left(\frac{g^{\prime}}{1 !}\right)^{k_{1}}\left(\frac{g^{\prime \prime}}{2 !}\right)^{k_{2}} \cdots\left(\frac{g^{(n)}}{n !}\right)^{k_{n}}$.

The formula is due to Francesco Faà di Bruno (see [1]) who lived from 1825 to 1888 and enjoys the rare (at least for mathematicians) distinction of being a Saint of the Catholic church (canonization in 1988 by Pope John Paul II). A proof using basic umbral calculus

Ableitungsregeln (etwa die Produktregel oder die Kettenregel) sind Formeln, die die Ableitung einer aus verschiedenen Einzelfunktionen zusammengesetzten komplizierteren Funktion durch die Ableitungen der Einzelfunktionen ausdrücken. Es liegt nahe, nach solchen Regeln auch für die höheren Ableitungen einer Funktion zu fragen. Die bekannte Leibnizsche Formel drückt etwa die höheren Ableitungen des Produktes zweier Funktionen durch die Ableitungen der einzelnen Faktoren aus. Eine - weit weniger bekannte - analoge Formel für die Verkettung zweier Funktionen wurde von dem italienischen Mathematiker Francesco Faà di Bruno entdeckt; für diese Formel wird in dem vorliegenden Artikel ein kurzer und elementarer Beweis angegeben. 
was given by Steven Roman in [3] where also references to other approaches can be found; a derivation using Hirzebruch's $m$-sequences is given in [4]. In this paper we present a completely elementary (and extremely short) proof which requires almost no prerequisites and allows the formula of Faà di Bruno to be incorporated into undergraduate calculus courses. (Some uses of the formula are given in [2].)

Proof. A trivial induction shows that there are polynomials $P_{n, k}$ (where $n$ is the number of variables of $P_{n, k}$ ) such that

$$
(f \circ g)^{(n)}=\sum_{k=0}^{n}\left(f^{(k)} \circ g\right) \cdot P_{n, k}\left(g^{\prime}, g^{\prime \prime}, \ldots, g^{(n)}\right)
$$

for all $f$ and $g$. In fact, the induction shows that these polynomials are recursively given by $P_{0,0}(x)=1$ and $P_{n+1, k}\left(x_{1}, \ldots, x_{n}, x_{n+1}\right)=x_{1} \cdot P_{n, k-1}\left(x_{1}, \ldots, x_{n}\right)+\sum_{i=1}^{n} x_{i+1}$. $\left(\partial_{i} P_{n, k}\right)\left(x_{1}, \ldots, x_{n}\right)$, if we interpret $P_{n, 0}$ and $P_{n, n+1}$ as zero, but this is irrelevant for our argument. What is important to realize from $(\star)$ is that $(f \circ g)^{(n)}\left(x_{0}\right)$ depends only on the values $g^{(k)}\left(x_{0}\right)$ and $f^{(k)}\left(g\left(x_{0}\right)\right)$ where $0 \leq k \leq n$; hence to establish the validity of the formula at any given point $x_{0}$, we may replace the given functions $f$ and $g$ with any functions $F$ and $G$ which have the same derivatives up to order $n$ as $f$ and $g$ at $g\left(x_{0}\right)$ and $x_{0}$, respectively. Hence, it suffices to prove the formula of Faà di Bruno for polynomials! Assuming $x_{0}=0$ and $g\left(x_{0}\right)=0$ without loss of generality, we may thus write $f(x)=a_{0}+a_{1} x+\cdots+a_{n} x^{n}$ and $g(x)=b_{1} x+b_{2} x^{2}+\cdots+b_{n} x^{n}$ where $a_{k}=f^{(k)}(0) / k$ ! and $b_{k}=g^{(k)}(0) / k$ ! for all $k$. In this case the formula to be proved reduces to the claim that the coefficient of $x^{n}$ in the expansion of $f(g(x))$ is

$$
\sum_{k=0}^{n} \sum_{\substack{k_{1}+k_{2}+\cdots+k_{n}=k, k_{1}+2 k_{2}+\cdots+n k_{n}=n}} \frac{k !}{k_{1} ! k_{2} ! \cdots k_{n} !} a_{k} b_{1}^{k_{1}} b_{2}^{k_{2}} \cdots b_{n}^{k_{n}} \text {. }
$$

But this is trivial! In fact, applying the multinomial formula

$$
\left(X_{1}+\cdots+X_{n}\right)^{k}=\sum_{k_{1}+\cdots+k_{n}=k} \frac{k !}{k_{1} ! k_{2} ! \cdots k_{n} !} X_{1}^{k_{1}} X_{2}^{k_{2}} \cdots X_{n}^{k_{n}}
$$

with $X_{k}:=b_{k} x^{k}$, we find

$$
\begin{aligned}
f(g(x)) & =\sum_{k=0}^{n} a_{k}\left(b_{1} x+b_{2} x^{2}+\cdots+b_{n} x^{n}\right)^{k} \\
& =\sum_{k=0}^{n} a_{k} \sum_{k_{1}+\cdots+k_{n}=k} \frac{k !}{k_{1} ! k_{2} ! \cdots k_{n} !} b_{1}^{k_{1}} b_{2}^{k_{2}} \cdots b_{n}^{k_{n}} x^{k_{1}+2 k_{2}+\cdots+n k_{n}} .
\end{aligned}
$$




\section{References}

[1] Faà di Bruno, F.: Traité Elementaire du Calcul. Gauthier-Villars, Paris 1869.

[2] Krantz, S.G.; Parks, H.R.: A Primer of Real Analytic Functions. Birkhäuser, Basel-Boston-Berlin 1992.

[3] Roman, S.: The Formula of Faà di Bruno. Amer. Math. Monthly 87 (1980), 805-809.

[4] Rabe von Randow: Über die Kettenregel n-ter Ordnung. Math. Ann. 192 (1971), 33-46.

Karlheinz Spindler

Fachbereich MNDU

Fachhochschule Wiesbaden

Kurt-Schumacher-Ring 18

D-65197 Wiesbaden, Germany

e-mail: spindler@r5.mnd.fh-wiesbaden.de 